\title{
Low temperature deactivation of Ge heavily n-type doped by ion implantation and laser thermal annealing
}

\author{
R. Milazzo, ${ }^{1}$ G. Impellizzeri, ${ }^{2}$ D. Piccinotti, ${ }^{1,3}$ D. De Salvador, ${ }^{1}$ A. Portavoce,${ }^{3}$ A. La Magna, ${ }^{4}$ \\ G. Fortunato, ${ }^{5}$ D. Mangelinck, ${ }^{3}$ V. Privitera, ${ }^{2}$ A. Carnera, ${ }^{1}$ and E. Napolitani ${ }^{1, a)}$ \\ ${ }^{1}$ CNR-IMM MATIS and Dipartimento di Fisica e Astronomia, Università di Padova, Via Marzolo 8, \\ 35131 Padova, Italy \\ ${ }^{2}$ CNR-IMM, Via S. Sofia 64, 95123 Catania, Italy \\ ${ }^{3}$ IM2NP, CNRS-Universités d'Aix-Marseille et de Toulon, Case 142, 13397 Marseille Cedex 20, France \\ ${ }^{4}$ CNR-IMM, Z.I. VIII Strada 5, 95121 Catania, Italy \\ ${ }^{5}$ CNR-IMM, Via del Fosso del Cavaliere 100,00133 Roma, Italy
}

(Received 4 August 2016; accepted 15 December 2016; published online 4 January 2017)

\begin{abstract}
Heavy doping of Ge is crucial for several advanced micro- and optoelectronic applications, but, at the same time, it still remains extremely challenging. Ge heavily n-type doped at a concentration of $1 \times 10^{20} \mathrm{~cm}^{-3}$ by As ion implantation and melting laser thermal annealing (LTA) is shown here to be highly metastable. Upon post-LTA conventional thermal annealing As electrically deactivates already at $350^{\circ} \mathrm{C}$ reaching an active concentration of $\sim 4 \times 10^{19} \mathrm{~cm}^{-3}$. No significant As diffusion is detected up to $450^{\circ} \mathrm{C}$, where the As activation decreases further to $\sim 3 \times 10^{19} \mathrm{~cm}^{-3}$. The reason for the observed detrimental deactivation was investigated by Atom Probe Tomography and in situ High Resolution X-Ray Diffraction measurements. In general, the thermal stability of heavily doped Ge layers needs to be carefully evaluated because, as shown here, deactivation might occur at very low temperatures, close to those required for low resistivity Ohmic contacting of n-type Ge. Published by AIP Publishing. [http://dx.doi.org/10.1063/1.4973461]
\end{abstract}

Due to its superior mobility of charge carriers that makes it able to attain higher drive currents, the interest in germanium is lately renewed with the aim of replacing silicon in future highly scaled Metal Oxide Semiconductor Field Effect Transistors (MOSFETs). ${ }^{1}$ However, a downscaling beyond $15-\mathrm{nm}$ necessarily requires doping levels as high as $1 \times 10^{20} \mathrm{~cm}^{-3}$ (Ref. 2) which, especially for $n$-type ultrashallow junctions, are still challenging due to the lower solid solubility and higher diffusivity of V-group elements. ${ }^{3-6}$ Heavy doping of $\mathrm{Ge}$ is also crucial for interesting applications in optoelectronics, such as lasing in the mid-IR range ${ }^{7}$ and mid-IR plasmon-enhanced sensing. ${ }^{8,9}$

The melting laser thermal annealing (LTA) technique has been recently considered in $\mathrm{Ge}^{10-12}$ as being the only technology able to activate dopants beyond their solid solubility limit. LTA also efficiently limits the diffusion processes, which are confined within the molten layer whose depth can be easily controlled by choosing the LTA energy density. ${ }^{2,13}$ In particular, As ion implantation followed by LTA has been recently demonstrated to be very promising for n-type doping, reaching an activation level up to $1 \times 10^{20} \mathrm{~cm}^{-3} \cdot 14-17$

However, the thermal stability of the obtained metastable heavily doped Ge layers has not been investigated so far, despite being a crucial aspect during further steps of device fabrication sequence. For example, it is worth considering that low resistivity n-type Ohmic contacts in Ge require germanidation processes at temperatures as high as $340^{\circ} \mathrm{C} .^{18}$

In this letter, we demonstrate that n-type Ge layers highly doped with As at a concentration of $1 \times 10^{20} \mathrm{~cm}^{-3}$ by ion implantation and melting LTA are highly metastable,

${ }^{a}$ Electronic mail: enrico.napolitani@unipd.it beginning to deactivate upon post-LTA conventional anneals already at $350{ }^{\circ} \mathrm{C}$, i.e., close to the above contacting temperatures. By combining Secondary Ion Mass Spectrometry (SIMS), Spreading Resistance Profiling (SRP), High Resolution X-Ray Diffraction (HRXRD), and Atom Probe Tomography (APT), we suggest that the As deactivation is correlated with a structural evolution and growth of As-V clusters.

A (100)-oriented Czochralski-Ge wafer has been implanted with $\mathrm{As}^{+}$ions at $40 \mathrm{keV}$ with a fluence of $3 \times 10^{15}$ $\mathrm{As} / \mathrm{cm}^{2}$, so as to obtain a maximum concentration of $7 \times 10^{20} \mathrm{As} / \mathrm{cm}^{3}$, i.e. well above the maximum equilibrium solubility $\left(8 \times 10^{19} \mathrm{As} / \mathrm{cm}^{3}\right) .{ }^{19}$ A single pulse or 10 pulses of melting LTA at $650 \mathrm{~mJ} / \mathrm{cm}^{2}$ have then been performed at room temperature (RT) and in vacuum with a Lambda Physik LPX $205 \mathrm{XeCl}$ excimer laser $(\lambda=308 \mathrm{~nm}$ and $28 \mathrm{~ns}$ pulse duration). As reported in Ref. 14, the ion implantation damage produces a surface amorphous layer $61 \mathrm{~nm}$ thick, and the crystal is fully recovered after the LTA process. The thermal stability has been investigated by performing conventional post-LTA thermal cycles in $\mathrm{N}_{2}$ atmosphere, consisting of a $100^{\circ} \mathrm{C} / \mathrm{min}$ linear ramp starting from RT up to the post-processing temperature that is kept constant for $10 \mathrm{~min}$ and a subsequent complete cooling phase down to RT. After each annealing cycle, the strain depth profiles have been measured in-situ by HRXRD; then, the samples have been heated again to higher temperatures, so as to probe the temperatures from 50 to $550{ }^{\circ} \mathrm{C}$, with steps of $50^{\circ} \mathrm{C}$. We recorded symmetrical (004) and, in selected samples, also asymmetrical (444) reciprocal lattice points. Since no mismatch in plane of the substrate was observed, we concluded that the doped layer is pseudomorphic in all the samples and focused only on the perpendicular strain, similarly to 
Ref. 17. For selected temperatures, the samples have been characterized also by SIMS, SRP, and APT. Concerning SRP, it is worth mentioning that the conversion of the spreading resistance data to the resistivity profiles was done by means of homogenously doped (n-type) Ge samples of known resistivity; the resistivity profiles were converted to carrier concentration profiles by means of the resistivity versus impurity concentration curve reported by Sze and Irvin. ${ }^{20}$

Figure 1 shows the As chemical (a), carrier (b), and strain (c) depth profiles (filled symbols) obtained in the $\mathrm{Ge}$ sample implanted with As after 10 pulses of LTA as measured by SIMS, SRP, and HRXRD, respectively. Figure 1(a) shows also the As chemical depth profile before the LTA

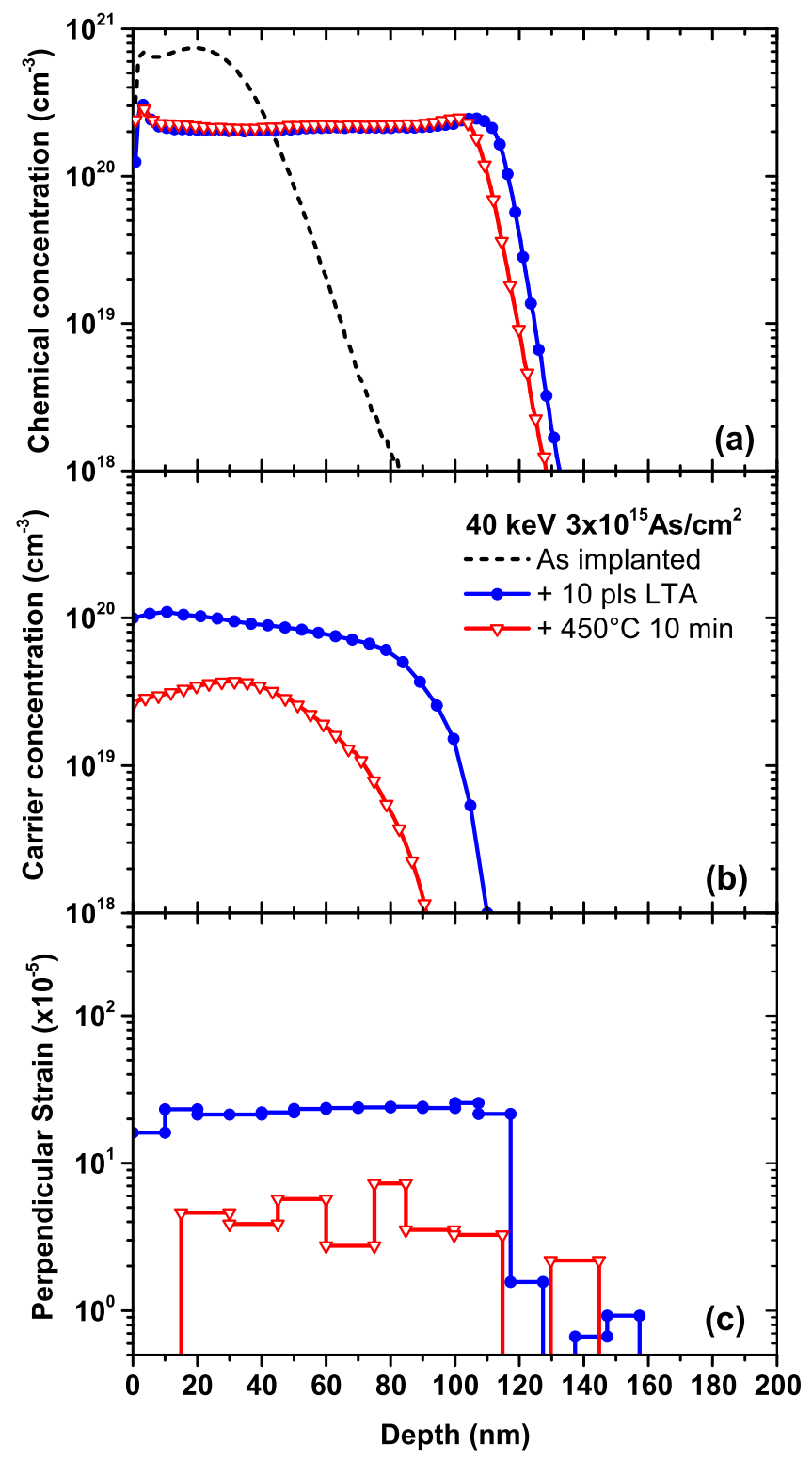

FIG. 1. Chemical (a), carrier (b), and perpendicular strain profiles (c) as measured by SIMS, SRP, and HRXRD, respectively, in Ge implanted with $3 \times 10^{15} \mathrm{As} / \mathrm{cm}^{2}$ at $40 \mathrm{keV}$, subsequently treated with 10 pulses of LTA at $650 \mathrm{~mJ} / \mathrm{cm}^{2}$ (circles), and post-LTA conventional annealing at $450{ }^{\circ} \mathrm{C}$ (triangles). The As concentration depth profile measured in the as implanted sample (dashed line) is also shown in panel (a). The strain at depths greater than $160 \mathrm{~nm}$ in panel (c) is compatible with zero in all the samples within $\pm 5 \times 10^{-6}$. Profiles after 10 pulses of LTA and before annealing are reproduced from Milazzo et al., J. Appl. Phys. 119(4), 045702 (2016). Copyright 2016 AIP Publishing LLC. process for comparison (dashed line). The box-like concentration profile observed after LTA (circles) is a characteristic feature of the multipulse processing, and it is due to the sharp drop of the diffusion coefficient at the maximum melt depth ( $\sim 120 \mathrm{~nm}$ for the used LTA conditions) when passing from the liquid to the crystalline phase. A remarkably high electrically active concentration of $1 \times 10^{20} \mathrm{~cm}^{-3}$ is also reached, which corresponds to approximately $50 \%$ of the total As concentration, as shown by Figure 1(b). The partial electrical activation after LTA is due to very small As-V clusters, as observed by positron annihilation spectroscopy measurements, ${ }^{21}$ most of them containing less than 5 As atoms, formed during the cooling transient of the LTA process. ${ }^{17}$ The positive strain reported in Figure 1(c) is due to both As atoms in substitutional, electrically active, configurations $\left(\mathrm{As}_{\mathrm{s}}\right)$ and As atoms in clusters $\left(\mathrm{As}_{\mathrm{cl}}\right)$, with lattice volume modification per atom of $\Delta \mathrm{V}_{\text {As_s }}=+8.8 \AA^{3}$ and $\Delta \mathrm{V}_{\text {As_cl }}=+2.0 \AA^{3}$, respectively. ${ }^{17}$

In order to evaluate the thermal stability, Figure 1 reports the same depth profiles after a further post-LTA conventional annealing cycle at $450^{\circ} \mathrm{C}$ (triangles). Despite the negligible As diffusion (the small shift in the maximum melt depth visible in Figure 1(a) is within the reproducibility of the LTA energy density between different spots), a significant deactivation is observed down to $\sim 3 \times 10^{19} \mathrm{As} / \mathrm{cm}^{3}$ (Figure 1(b)). It is worth noting that this carrier level is lower than the active concentration obtained with non-melting annealing processes of $5 \times 10^{19} \mathrm{As} / \mathrm{cm}^{3}{ }^{22}$ Thus, the postLTA thermal process suppresses all the advantages of the LTA in terms of electrical activation. Consistently, a significant reduction of the strain level is also observed upon postLTA annealing, as shown in Figure 1(c).

With the aim of describing the evolution of the deactivation mechanism we extracted, from the same sample reported in Figure 1, the chemical and the carrier concentration as well as the perpendicular strain at the intermediate depth of $\sim 20 \mathrm{~nm}$ for different post-LTA cycles from 50 to $550^{\circ} \mathrm{C}$, as reported in Figure 2. The chemical concentration remains unchanged up to $450^{\circ} \mathrm{C}$, consistent with Figure 1, while a significant reduction is evidenced at $550{ }^{\circ} \mathrm{C}$ due to a significant As diffusion as evidenced by SIMS (not shown). On the contrary, the carrier concentration remains almost constant up to $250^{\circ} \mathrm{C}$ and then significantly drops at $350{ }^{\circ} \mathrm{C}$

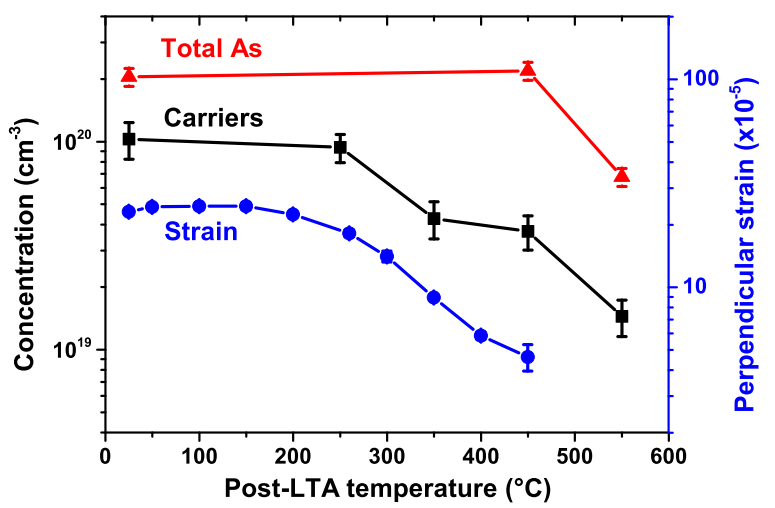

FIG. 2. Arsenic chemical concentration (triangles), carrier concentration (squares), and perpendicular strain (circles) as extracted at the depth of $20 \mathrm{~nm}$ by SIMS, SRP, and HRXRD, respectively, in Ge implanted with $3 \times 10^{15} \mathrm{As} / \mathrm{cm}^{2}$ at $40 \mathrm{keV}$, after LTA with 10 pulses at $650 \mathrm{~mJ} / \mathrm{cm}^{2}$ and post-LTA conventional thermal cycles for several temperatures. 
$\left(4 \times 10^{19} \mathrm{As} / \mathrm{cm}^{3}\right)$, whereas only a slight further reduction is observed at $450{ }^{\circ} \mathrm{C}$. At $550^{\circ} \mathrm{C}$, there is a significant additional reduction of the active concentration, but this is ascribed to the broadening of the As depth distribution. The perpendicular strain shows an almost constant value up to $200{ }^{\circ} \mathrm{C}$, and then, a monotonic decrease occurs by increasing the temperature qualitatively consistent with the carrier concentration. A similar behavior is observed in samples subjected to a single LTA pulse (not shown). It is worth noting that these temperatures are particularly low in view of device applications, especially if it is considered that low resistance contacting requires anneals at around $340^{\circ} \mathrm{C}$. $^{18}$

The strain reduction observed in Figure 2 is due to the As atoms which, as a result of the deactivation process, move from substitutional to cluster sites, where they induce a lower lattice volume modification per atom (we recall ${ }^{17}$ that $\Delta \mathrm{V}_{\mathrm{As} \_\mathrm{s}}=+8.8 \AA^{3}$ and, in the case of clusters formed during LTA, $\left.\Delta \mathrm{V}_{\mathrm{As} \_\mathrm{cl}}=+2.0 \AA^{3}\right)$. However, as the As offlattice sites still retain some positive strain $\left(\Delta \mathrm{V}_{\mathrm{As} \_c l}\right.$ is about one fourth of $\Delta \mathrm{V}_{\text {As_s }}$ ) whereas they do not provide carriers anymore, the strain is expected to decrease along with the temperature more slowly than the carrier concentration if the above $\Delta \mathrm{V}_{\text {As_s }}$ and $\Delta \mathrm{V}_{\text {As_cl }}$ values are assumed. Surprisingly, Figure 2 shows instead that the strain decreases slightly faster than the carrier concentration (the two quantities have been reported in the figure with identical log scales).

Given that substitutional As has a fixed and well-defined configuration, it is straightforward to assume that $\Delta \mathrm{V}_{\mathrm{As}_{\_} \mathrm{s}}$ is constant, irrespective of the annealing process. Therefore, in order to explain the above behavior, it can be concluded that the lattice volume modification per As atom in clusters formed after post-LTA processes must reduce with respect to As in clusters formed after LTA processes, thus suggesting that the post-LTA As deactivation is correlated with a structural evolution of the As atoms in clusters. In order to quantify such a behavior, Figure 3 reports the $\Delta \mathrm{V}_{\mathrm{As} \_\mathrm{cl}}$ as calculated from the measured values of the perpendicular strain and the As chemical and carrier concentrations and by assuming $\Delta \mathrm{V}_{\mathrm{As} \_\mathrm{s}}=+8.8 \AA^{3}$ (see Ref. 17 for details on the

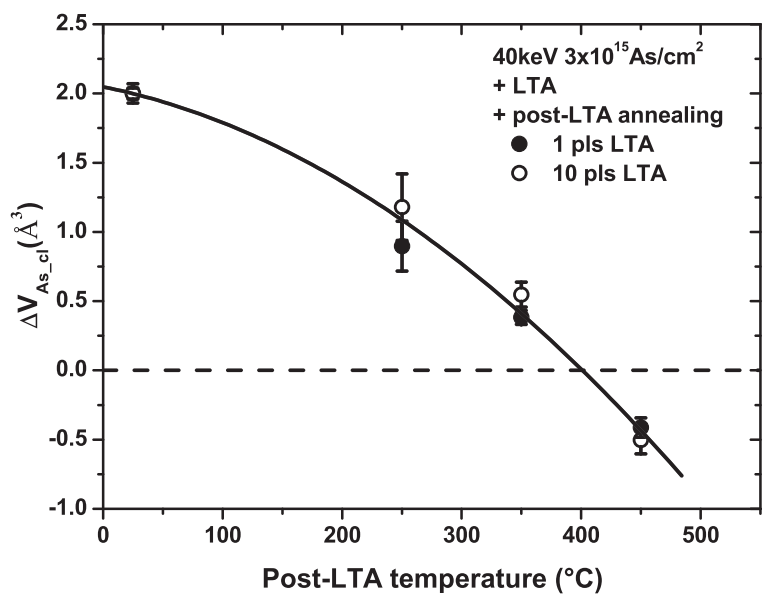

FIG. 3. Lattice volume modification per $\mathrm{As}$ atom in clusters $\Delta \mathrm{V}_{\mathrm{As} \text { _cl }}$ extracted in Ge samples implanted with $3 \times 10^{15} \mathrm{As} / \mathrm{cm}^{2}$ at $40 \mathrm{keV}$, after LTA with 1 pulse (filled circles) or 10 pulses (open circles) at $650 \mathrm{~mJ} / \mathrm{cm}^{2}$ and further post-LTA conventional thermal cycles for several temperatures. The data at $25^{\circ} \mathrm{C}$ refer to the sample after LTA. The continuous line has been drawn to guide the eye. extraction of this value from the measured quantities), as a function of the post-LTA annealing temperature.

As can be seen from Figure $3, \Delta \mathrm{V}_{\text {As_cl }}$ decreases by increasing the post-LTA temperature from the initial positive value of $\Delta \mathrm{V}_{\mathrm{As} \_\mathrm{cl}}=+2.0 \AA^{3}$ recorded after $\mathrm{LTA}^{17}$ down to the negative value of $-0.5 \AA^{3}$. Even if the HRXRD data cannot provide direct evidence of cluster evolution, the $\Delta \mathrm{V}_{\mathrm{As} \_\mathrm{cl}}$ reduction and the reversal reported in Figure 3, i.e., the reduction of the lattice expansion related with As and the eventual lattice contraction, suggest that the As deactivation is related to the trapping of vacancies by the As clusters, so that the number of vacancies per As cluster increases by increasing the temperature, similar to what is observed for the As doping of silicon. ${ }^{23}$ We speculate that the As deactivation is driven by $\mathrm{V}$ point defects, thermally generated and/ or residual from the LTA process, which interact with substitutional As. The resulting AsV mobile species is then trapped by As-V clusters already nucleated after $\mathrm{LTA}^{21}$ and, at the same time, reaching a clustered, inactive site altering the configuration of the existing clusters. This scenario is consistent with Density Functional Theory (DFT) calculations that report an unfavorable As-As binding energy in $\mathrm{Ge}$, which becomes more stable if mediated by interaction with vacancies. ${ }^{24}$

In order to gain further insights on the above As deactivation mechanisms, we also performed APT analyses on the two samples discussed in Figure 1, i.e., those before and after post-LTA cycles up to $450^{\circ} \mathrm{C}$. From the 3D As distribution obtained by APT, we extracted the cluster count distribution as a function of the number of As atoms per cluster (a lower cut-off of 5 atoms/cluster has been considered in order to exclude false clusters) through a statistical analysis (for more details, see Ref. 25). Then, we normalized the cluster count distribution to the volume analyzed by APT, obtaining the cluster density as a function of the number of As atoms per cluster, as reported in Figure 4. A remarkable shift toward larger As clusters can be noticed, hence indicating their size is growing along with the As deactivation. Furthermore, the size of the biggest clusters increases from 8 to 10 atom/ cluster after LTA to 25-30 atoms/clusters after post-LTA. This large increase indicates that, concurrently with the

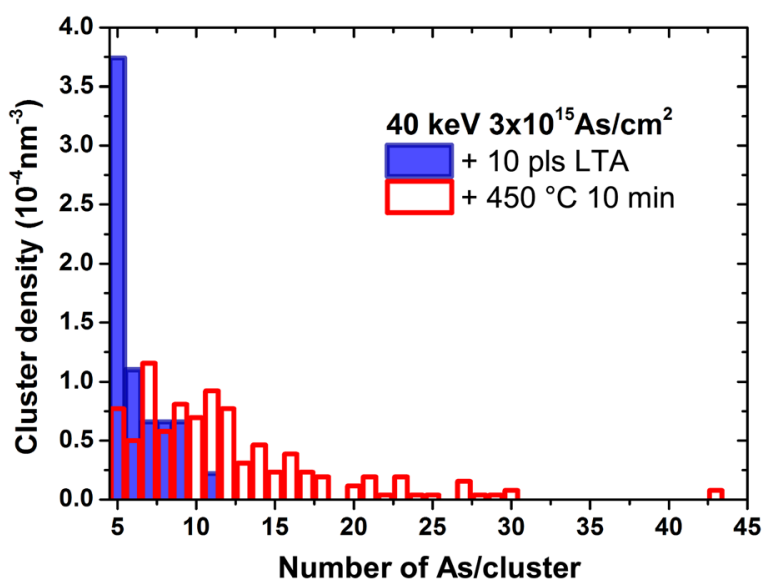

FIG. 4. Cluster density as a function of the number As atoms per cluster as extracted by statistical analysis of APT 3D As distributions measured on samples implanted with $3 \times 10^{15} \mathrm{As} / \mathrm{cm}^{2}$ at $40 \mathrm{keV}$, annealed by LTA with 10 pulses at $650 \mathrm{~mJ} / \mathrm{cm}^{2}$ (full columns), and subjected to further post-LTA conventional annealing at $450{ }^{\circ} \mathrm{C}$ (open columns). 
deactivation mechanism discussed previously which increases the population of clustered As, an Ostwald ripening growth mechanism also occurs, favoring the growth of more stable bigger clusters with respect to the less stable smaller ones. Unfortunately, due to the threshold of 5 atoms per cluster, the distributions reported in Figure 4 represent a fraction of only a few percent of the inactive As atoms. Thus, a detailed experimental description of the cluster evolution is not feasible, demanding for future more detailed modeling of the experimental data.

In conclusion, we demonstrated that conventional postLTA thermal processing promotes the electrical deactivation of As in heavily doped Ge. The electrical deactivation begins at the relatively low temperature of $350{ }^{\circ} \mathrm{C}$ reaching an active concentration of $\sim 4 \times 10^{19} \mathrm{As} / \mathrm{cm}^{2}$, and the active As concentration further decreases down to $\sim 3 \times 10^{19} \mathrm{As} / \mathrm{cm}^{2}$ at $450{ }^{\circ} \mathrm{C}$ while maintaining negligible As diffusion. The origin of the As deactivation is ascribed to diffusion and clustering of substitutional As promoted by $\mathrm{V}$ point defects, accompanied with a cluster growth through an Ostwald ripening mechanism. These results reveal that the beneficial role of LTA in Ge in terms of achieving high As doping levels is lost after moderate post-LTA anneals. In general, this constitutes a warning against the high metastable nature that heavily doped n-type layers can have in Ge. In fact, we cannot exclude that, similarly to what is shown here for As, heavy n-type doping with other species such as $\mathrm{P}$ or $\mathrm{Sb}$ might also easily deactivate at temperatures comparable to those required for low resistance Ohmic contacting, which can be detrimental for several advanced applications of $\mathrm{Ge}$.

The authors wish to thank R. Storti and L. Bacci (University of Padova) for their technical expert assistance and M. Italia (CNR-IMM Catania) for the SRP measurements. The authors acknowledge financial support from the French CNRS (FR3507) and CEA METSA network (www.metsa.fr).

${ }^{1}$ R. Pillarisetty, Nature 479, 324-328 (2011).

${ }^{2}$ See http://www.itrs.net for ITRS, 2013 edition.

${ }^{3}$ A. Chroneos and H. Bracht, Appl. Phys. Rev. 1, 011301 (2014).

${ }^{4}$ R. G. Elliman and J. S. Williams, Curr. Opin. Solid State Mater. Sci. 19(1), 49-67 (2015).

${ }^{5}$ E. Napolitani and G. Impellizzeri, Defects in Semiconductors (Elsevier/ Academic Press, 2015), pp. 93-122.
${ }^{6}$ G. Impellizzeri, S. Boninelli, F. Priolo, E. Napolitani, C. Spinella, A. Chroneos, and H. Bracht, J. Appl. Phys. 109, 113527 (2011).

${ }^{7}$ J. Liu, X. Sun, R. Camacho-Aguilera, L. C. Kimerling, and J. Michel, Opt. Lett. 35, 679-681 (2010).

${ }^{8}$ J. Frigerio, A. Ballabio, G. Isella, E. Sakat, G. Pellegrini, P. Biagioni, M. Bollani, E. Napolitani, C. Manganelli, M. Virgilio, A. Grupp, M. P. Fischer, D. Brida, K. Gallacher, D. J. Paul, L. Baldassarre, P. Calvani, V. Giliberti, A. Nucara, and M. Ortolani, Phys. Rev. B 94, 85202 (2016).

${ }^{9}$ L. Baldassarre, E. Sakat, J. Frigerio, A. Samarelli, K. Gallacher, E. Calandrini, G. Isella, D. J. Paul, M. Ortolani, and P. Biagioni, Nano Lett. 15, 7225-7231 (2015).

${ }^{10}$ G. Impellizzeri, E. Napolitani, S. Boninelli, G. Fisicaro, M. Cuscunà, R. Milazzo, A. La Magna, G. Fortunato, F. Priolo, and V. Privitera, J. Appl. Phys. 113, 113505 (2013).

${ }^{11}$ S. Heo, S. Baek, D. Lee, M. Hasan, H. Jung, J. Lee, and H. Hwang, Electrochem. Solid State Lett. 9(4), G136-G137 (2006).

${ }^{12}$ E. Bruno, G. G. Scapellato, A. La Magna, M. Cuscunà, E. Napolitani, S. Boninelli, F. Priolo, G. Fortunato, and V. Privitera, Appl. Phys. Lett. 101, 172110 (2012).

${ }^{13}$ J. M. Poate and J. W. Mayer, Laser Annealing of Semiconductors (Academic Press, New York, 1982).

${ }^{14}$ R. Milazzo, E. Napolitani, G. Impellizzeri, G. Fisicaro, S. Boninelli, M. Cuscunà, D. De Salvador, M. Mastromatteo, M. Italia, A. La Magna, G. Fortunato, F. Priolo, V. Privitera, and A. Carnera, J. Appl. Phys. 115, 053501 (2014).

${ }^{15}$ M. Shayesteh, D. O. Connell, F. Gity, P. Murphy-Armando, R. Yu, K. Huet, I. Toque-Tresonne, F. Cristiano, S. Boninelli, H. H. Henrichsen, P. F. Nielsen, D. H. Petersen, and R. Duffy, IEEE Trans. Electron Devices 61, 4047-4055 (2014).

${ }^{16}$ R. Milazzo, G. Impellizzeri, M. Cuscunà, D. De Salvador, M. Mastromatteo, A. La Magna, G. Fortunato, F. Priolo, V. Privitera, A. Carnera, and E. Napolitani, Mater. Sci. Semicond. Process 42, 196-199 (2016).

${ }^{17}$ R. Milazzo, G. Impellizzeri, D. Piccinotti, A. La Magna, G. Fortunato, D. De Salvador, A. Carnera, A. Portavoce, D. Mangelinck, V. Privitera, and E. Napolitani, J. Appl. Phys. 119(4), 045702 (2016).

${ }^{18}$ K. Gallacher, P. Velha, D. J. Paul, I. MacLaren, M. Myronov, and D. R. Leadley, Appl. Phys. Lett. 100, 22113 (2012).

${ }^{19}$ V. I. Fistul, Impurities in Semiconductors: Solubility, Migration, and Interactions (CRC Press, Florida, 2004), p. 157.

${ }^{20}$ S. M. Sze and J. C. Irvin, Solid State Electron. 11, 599 (1968).

${ }^{21}$ T. Kalliovaara, J. Slotte, I. Makkonen, J. Kujala, F. Tuomisto, R. Milazzo, G. Impellizzeri, G. Fortunato, and E. Napolitani, Appl. Phys. Lett. 109, 182107 (2016).

${ }^{22}$ G. Hellings, E. Rosseel, E. Simoen, D. Radisic, D. Hjorth Petersen, O. Hansen, P. Folmer Nielsen, G. Zschatzsch, A. Nazir, T. Clarysse, W. Vandervorst, Th. Y. Hoffmann, and K. De Meyer, Electrochem. Solid State Lett. 14, H39 (2011).

${ }^{23}$ G. Borot, L. Rubaldo, L. Clement, R. Pantel, D. Dutartre, K. Kuitunen, J. Slotte, F. Tuomisto, X. Mescot, M. Gri, and G. Ghibaudo, J. Appl. Phys. 102, 103505 (2007).

${ }^{24}$ A. Chroneos, R. W. Grimes, B. P. Uberuaga, S. Brotzmann, and H. Bracht, Appl. Phys. Lett. 91, 192106 (2007).

${ }^{25}$ L. T. Stephenson, M. P. Moody, P. V. Liddicoat, and S. P. Ringer, Microsc. Microanal. 13, 448-463 (2007). 